\title{
The use of online videos in the 2008 US congressional elections
}

\author{
Eni Mustafaraj, Panagiotis Takis Metaxas, and Catherine Grevet \\ Department of Computer Science \\ Wellesley College \\ Email: (emustafa, pmetaxas, cgrevet)@wellesley.edu
}

\begin{abstract}
With 39\% of Americans admitting the use of the Web to get unfiltered campaign materials, it becomes important to evaluate how they are searching for these materials and what they are finding. Assuming that the search will take place on one of the major search engines, such as Google, the results need to be scrutinized to ensure that standards of fairness and balanced coverage are upheld. In this paper, we offer an exploratory analysis of political online video data collected in the framework of a broader project aimed at capturing efforts of spamming search engine results for political motives. By exploiting online video features such as the added date, number of views, and ranking position, as well as content related features such as description keywords, political inclination of the submitter, the political message, and comments associated with a video, we depict a picture of how the online video medium was used during the last congressional political campaign. Our analysis takes into account three players: video providers (usually the campaigns or other interested parties), video consumers (the users), and facilitators (Google and YouTube). The results show that online video coverage might be susceptible to technological bias that adds to the political bias common in electoral campaigns. Educating the wide audience of users about this inherent bias should be a common effort of the involved players and fairness advocacy groups.
\end{abstract}

\section{INTRODUCTION}

The last report "The Internet and the 2008 Election" [1], published by Pew Internet \& American Life Project, found that " $35 \%$ of Americans have watched online political videos", while "10\% have used social networking sites such as Facebook or MySpace to gather information or become involved". The fact that so many Americans have watched online political videos should not be surprising when taking into account that YouTube, the online video sharing website, ranks globally at the 3rd position behind Yahoo! and Google in terms of traffic [source: alexa.com]. Although YouTube was established only in 2005, its power as a new political medium was displayed during the 2006 Congressional elections, when a posted video-clip containing a controversial racial remark of the incumbent republican Senator of Virginia, George Allen, seeking reelection, was turned into a national phenomenon, probably becoming one of the reasons Allen lost his up-tothat-moment secure seat [3]. After that episode, none of the political candidates running for office could afford to ignore the far-reaching power of online video. In fact, the 2008 US Presidential elections showed the many different ways online video can damage a candidate, starting with the video-clips of the former pastor of Barack Obama, Jeremiah Wright, and ending with the interviews of Sarah Palin and her ridiculing impersonations. However, many candidates tried to use the medium at their advantage, by establishing YouTube channels where they regularly uploaded their video ads, speeches, debates, and other moments from the campaign. For example, BarackObamadotcom channel has more than 1800 videos and JohnMcCaindotcom has 330 videos.

Although many users directly access YouTube in search for videos, the majority starts the search for information from the search box of a search engine, such as Google [2]. Consequently, if a user is searching about a political candidate, the results produced by the search engine could have an impact on the opinion the user forms about the candidate. Moreover, it is known that search engine results can be manipulated. In 2006, news publications reported that political blogs had been actively trying to influence the US elections by pushing web pages carrying negative content to the top of the relevant search results of the major search engines. This practice of "gaming" the search engines was implemented with link bombing techniques (also known as Googlebombing), in which web site masters and bloggers use the anchor text to associate an obscure, negative term with a public entity [4]. In particular, during the 2006 US midterm congressional election, a concerted effort to manipulate ranking results in order to bring to public attention negative stories about Republican incumbents running for Congress took openly place under the solicitation of the progressive blog, MyDD.com (My Direct Democracy) [5]. Therefore, detecting efforts of gaming the search results by third parties is important in the light of evaluating fairness in the public political discourse. We have collected search results about candidates in the 2008 US Congressional elections during a six months period, with the purpose of studying Googlebombing as well as the response of search engines to such attacks. Some of the study findings are shortly discussed in Section II and can be found fully in [6]. During the data collection process for that study, described in Section III, we noticed that YouTube results are very common in Google search results and posed ourselves the following research questions:

1) Who are the video providers and what kind of videos they provide?

2) How are users consuming these political videos?

3) What kind of a role do Google and YouTube play in 
the process of connecting video providers with video consumers?

For our exploratory analysis, we used a series of quantitative and qualitative features to answer these questions and the results are presented in Section IV. Based on our findings, we discuss the question of inherent technological bias in Section $\mathrm{V}$ and conclude with ideas for future work in Section VI.

\section{Motivation}

Another result of the previously-mentioned Pew report is that $39 \%$ of Americans have used Internet to access "unfiltered" campaign materials during the 2008 primary elections. The term "unfiltered" however might be misleading. Users believe that traditional media outlets, by filtering their coverage of the candidates based on their political bias, are limiting users' exposure to relevant information, and therefore the Web is a most reliable medium to access that information. The key issue here is that not many users know how "relevance" is defined by search engines or other web applications (such as YouTube) that serve as gatekeepers to the huge amount of unfiltered information on the web. Because most users do not click beyond the links displayed in the first page of results, what appears on the first page is very important. As previously mentioned, a gaming effort such as Googlebombing was successful during the 2006 US midterm elections. This motivated us to study whether such a gaming effort would take place again in 2008 and what Google was doing to prevent it.

Because it is difficult to analyze Googlebombing after it has become public, we decided to perform systematic data collections well in advance of the 2008 US Congressional elections. A a time when the attention of the public and the mainstream media was focused on the historical presidential race, we focused our attention on the most contested races for Congress. A detailed analysis of our findings appears in [6], and in summary we can say that we did not encounter Googlebombing efforts such as those of 2006. In fact, averaged over 24 weeks, almost $70 \%$ of the top-5 search results belonged either to the official campaign site of a candidate, to the official congressional site (in case of an elected official), or to the candidate's Wikipedia entry. These results remained stable over time.

In the process of our data analysis, we noticed that YouTube videos appeared frequently in the search results (about $4 \%$ of all links, averaging around 2 links per candidate). Because videos have the potential to be used by partisan groups to spread political messages, and because especially during this electoral cycle there was an increased awareness about online video material, we decided in a second phase to analyze data related to video usage. Since the data were not collected with the original intention to study online video usage during the electoral campaign, our results might not be comprehensive. However, our analysis offers insights that can be used in designing a dedicated experiment for a more accurate evaluation.

\section{Methodology}

During the six month period June - December 2008, we collected search results delivered by Google for the names of more than 100 candidates competing for seats in the House of Representatives and the Senate once a week. More exactly, we followed 59 contested races, 48 for the House and 11 for the Senate, although the names of a few candidates were missing at the start of the data collection phase. This was because some districts were holding their primaries to decide the candidates after June 2008. The decision on what races to follow was based on collected information from several websites such as: The Electoral Vote Predictor (www.electoral-vote.com), Open Secrets (openSecrets.org) maintained by the nonpartisan group "The Center for Responsive Politics", the Democratic Congressional Campaign Committee (www.dccc.org), and the National Republican Congressional Committee (www.nrcc.org).

\section{A. Google Data}

Google provides an API (Application Programming Interface) to automatically access its search index. We issued every candidate name as a phrase (i.e., inside quotes) to the Google Search API and stored the top 20 returned URLs. Results look like the following, for the candidate Norm Coleman:

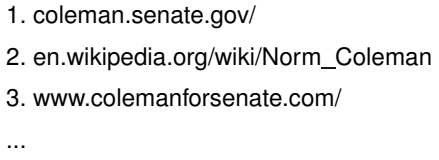

Repeating the same queries every week enabled us to record the ups and downs of different URLs in the list and to discern when a URL entered or left the Top 10 (the first page of Google results).

After collecting these links, a script fed each URL to an HTTP request to get and store locally the corresponding HTML page. We have complete data for 105 candidates known in June 2008, and partial data for 13 candidates added in September 2008.

\section{B. YouTube Data}

There are several sources on the Web where it is possible to watch online videos: news channels such as CNN and FoxNews, daily newspapers such as New York Times and Wall Street Journal, blogs, and social networking websites such as Facebook and MySpace. However, because video is embedded within the HTML webpage, the only way to know that one of these pages contains video material is by inspecting its HTML source code. In contrast, a URL starting with youtube.com immediately identifies an online video. Because of this simplicity in spotting online videos without having to parse HTML code from different websites, the data used in our analysis belong all to the YouTube website. Thus, in the context of this paper "online video" and "YouTube video" are equivalent expressions.

By storing every week the top 20 URLs returned by Google for every candidate name, we collected a set of almost 65,000 
TABLE I

FeAtures of A Youtube VIDEO

\begin{tabular}{|l|l|}
\hline Feature & Description \\
\hline \hline Video ID & An 11-character long string to uniquely identify a video. \\
\hline From & The username of the entity that uploaded the video. \\
\hline Title & A short title for the video. \\
\hline Keywords & A list of comma-separated labels for the video. \\
\hline Description & A short description of the video content. \\
\hline Category & Video category such as Politics, News, Music, etc. \\
\hline Added & The date on which video was added on YouTube. \\
\hline Views & The number of times a video is viewed. \\
\hline Ratings & The number of times a video is rated by viewers. \\
\hline Comments & The number of times a video is commented by viewers. \\
\hline Favorite & The number of times a video is favored by viewers. \\
\hline
\end{tabular}

URLs. We filtered from this set all URLs containing the string "youtube", receiving a subset of almost 2,500 URLs. Because retrieving HTML pages from a set of URLs over time is not always a successful process, our script had stored only 2,128 HTML pages in the server.

\section{Video Features Used for Analysis}

The stored HTML files for every YouTube video can be parsed to extract several pieces of information that allow the analysis of the video and its usage. Table I contains a list of the features we extracted for each video. The feature names are the same ones used to identify these features in the HTML code.

One of the most important features that allows to perform an accurate analysis is the video id. Although Google returns unique URLs in its results, often a URL contains extra information. Thus, while in the list of 2,500 URLs there are 450 unique URLs, these represent only 406 unique videos. More exactly, there are 398 videos and 8 video channels. A video channel is a URL such as: youtube.com/user/ JeanneShaheen 2008 , while a simple video is a URL such as: www youtube. $\mathrm{com} /$ wat $\mathrm{ch}$ ? $\mathrm{v}=\mathrm{qI}-\mathrm{mq} 8 \mathrm{uN} 6 \mathrm{NI}$. Since a video chanel contains several video (even hundreds), we did not included them in our analysis.

\section{ANALYSIS}

We analyzed the video data from three perspectives, in order to determine the role of video providers, video consumers, and video facilitators.

\section{A. Video Providers}

The first question we posed was who the video providers are, and what kind of content they are providing. As a video provider, we refer to the YouTube registered user, who has uploaded a video on YouTube. A video provider is not necessarily the content creator. We are not interested in who created the video, but in who decided to make the video available to others. The provider username is determined by the "From" feature.

Initially, we analyzed the content of videos by reading their metadata descriptions and by watching the videos. As a result of this process we divided the videos in two groups: (A) relevant to the candidates for election (366), and (B) irrelevant to the candidates (videos belonging to other people with the same name, or lost videos) (32). The videos of group (A) were further subcategorized according to the type of provider: a) the candidate's campaign; b) a political group; c) other (neither a campaign nor a group), and according to the political message: a) pro candidate, b) against candidate, c) neutral to candidate. The distribution of videos according to these two categories is summarized in Table II.

The numbers in Table II show that the political groups are the most frequent video providers with a total of 173 videos, followed by the candidates with 120 videos, and others with 73 videos. Many of the providers had uploaded more than one video. The most prolific were DCCCLive (the Democratic Congressional Campaign Committee), with 54 videos, and TheNRSC (the National Republican Senatorial Committee), with 16 videos. There were in total 200 different video providers. According to the type of provider, there were 77 groups, 63 others, and 60 candidates.

TABLE II

VIDEO DISTRIBUTION ACROSS TWO CATEGORIES

\begin{tabular}{|l|r|r|r|}
\hline \multirow{2}{*}{ Provider } & \multicolumn{3}{|c|}{ Political Message } \\
\cline { 2 - 4 } & Pro & Against & Neutral \\
\hline Candidate & 109 & 12 & - \\
\hline Group & 31 & 123 & 18 \\
\hline Other & 26 & 29 & 18 \\
\hline
\end{tabular}

1) Pro and against videos: In the group of 366 relevant videos, we found 165 video with a pro-candidate message, 165 video with an against-candidate message, and 36 videos with a neutral message. We also categorized videos based on the format of their content: a) ads, b) non-ads. We found 207 ads and 159 non-ads. The majority of ads (123) were negative ads. As the Table II shows, the candidates themselves have rarely engaged in negative campaigning (a direct attack to their opponent), there are only 11 such videos. This task was frequently performed by their parties at the national or regional level. Concretely, DCCCLive had 52 negative videos out of a total of 54, and TheNRSC had 16 negative videos out of 16. Especially the large number of negative ads shows that YouTube is regarded by the video providers similar to TV. The same 30 seconds long, negative, made-for-TV ads that are also uploaded on YouTube, since such a service is free.

2) Strategic timing: Using the "Added" feature of each video, which indicates the complete date on which a video was uploaded to YouTube, we created the graph shown in Figure 1. The three plotted lines belong to the years 2006, 2007, and 2008. It should be noted that in 2006 and 2008, congressional elections took place in November. The graph shows that in both these years, the highest activity belongs to the period September-October, right before the elections.

By comparing the date at which a video was added on YouTube with the date at which that video entered the top 20 URLs produced by the Google Search index, we calculated 
the average number of days needed to break into the top 20 . The numbers (for the year 2008) are the following: June28,6 days; July -29 days; August-30,9 days; September14,2 days; October-14,3 days. This short period of time to enter top 20 for the months of September and October might indicate two things: users are more engaged with watching video when the election date draws nearer, Google crawls more often YouTube results in the election season and includes results earlier in the ranking.

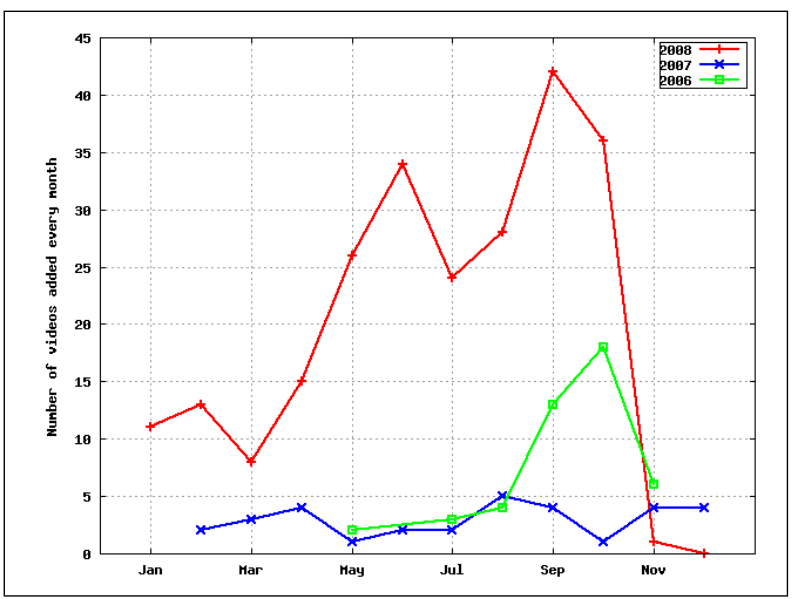

Fig. 1. Distribution of the number of YouTube videos according to their added date.

\section{B. Video Consumers}

There are several features that indicate how users are consuming videos: the number of views, the number of ratings and the average rating, the number of comments and what they are saying, or the number of selecting a video as a favorite. In this analysis we will discuss viewership and comments.

1) Viewership: Uploading videos on YouTube does not tantamount to attracting a large viewership. At least this is true for the majority of the candidates. There are however clear outliers. Senator Ted Stevens with his two videos described in Table III was the most successful candidate, maybe because his videos have nothing to do with his political message. A second best is the comedian-turned-politician Al Franken, and again the reason has not to do with his quite recent political carrier. By using the Views feature of the videos, we calculated the number of times a video was watched during the period it appeared in our data collection (we subtracted from the views count of the last entry the views count of the first entry in the data corpus). The results showed 5 videos from Stevens and Franken with more than 100,000 views per video, 5 videos with 10,000 to 40,000 views per video, 28 videos with 1,000 to 10,000 views, and the rest of videos with less than 1,000 views. Dividing the most watched 100 videos according to their political message, we found that $54 \%$ belong to negative videos, $29 \%$ to positive videos, and $17 \%$ to neutral videos. Since our data set has an equal number of negative and positive videos, these percentages show that users prefer to watch negative videos.
2) Comment Analysis: Given the debate nature of politics, it would be expected that videos in our sample data would spark large discussions. Surprisingly, it appeared that a very small fraction (less than $5 \%$ ) of viewers posted a comment per video, and it may be even less if some users posted more than one comment per view. This may be explained by the fact that YouTube videos can easily be embedded on other webpages such as blogs or social networking websites and the political discussion may happen on those sites.

To understand why some videos receive comments while others don't, we could analyze the content of the comments to reveal trends. This is difficult due to semantics misinterpretations and language subtleties. However, it is possible to extract a high-level categorization such as comments on the quality of the video: "That is probably one of the worst advertisements I've ever seen."1, asking other viewers for input: "Any fallout on this? Can we expect a follow up?"2, agreeableness with video content: "hell yes, i agree!"3, additional facts to provide more context for video: "The volunteer happens to also be the spouse of one of Ashwin Madia campaign staffers"4, and tangential comment or irrelevant comments: "I swear to God, all the liberals drive Subaru's." . Taking this categorization into account could measure certain user characteristics such as dislike, disagreement, or confusion. This exploration constitutes a framework for future work.

\section{Video Facilitators}

In our study we defined as video facilitators YouTube-as the platform were videos are stored and consumed, and Googleas the search engine were YouTube videos can be found as part of the organic search results displayed to a user when searching for a candidate. However, the reader is reminded that YouTube is a subsidary of Google Inc.

1) Relevance bias: How does Google decide which YouTube video to rank higher in the search results? Compare the data for the two videos shown in Table III.

During the first month of our data collection (June 2008), Video_B ranked always in the 4th position, and Video_A ranked always in the 5th position. However, after June, Video_B continued to remain constantly in the top 10 (overall average ranking: 6), while Video_A fell out of the top 10 and in October even out of the top 20. During all the time that Video_A was in the ranking, it had approximately 3 times more views per week than Video_B. And the overall statistics show that although both videos were added on YouTube at the same time, Video_A had more viewers, more ratings, and was more favored by the viewers. So, why it disappeared from the top 20 a month before the elections? We don't have an answer for that, but we point out to the different nature of the two videos. Video_A ridicules the famous speech on

\footnotetext{
${ }^{1}$ Mark Warner: Join our effort

${ }^{2}$ Ashwin Madia Volunteer Caught on Film Stealing Paulsen for Congress Lawn Signs

${ }^{3}$ Former VA Gov. Mark Warner's DNC Speech

${ }^{4}$ See footnote 2 .

${ }^{5}$ See footnote 2 .
} 
TABLE III

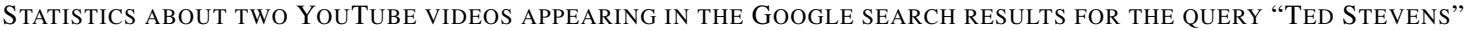

\begin{tabular}{|l||r|r|}
\hline & Video_A: DJ Ted Stevens Techno Remix: “A series of tubes” & Video_B: Series of tubes \\
\hline \hline Views at start date & $3,054,663(\mathrm{Jun}$ 9, 2008) & 297,064 (Jun 09, 2008) \\
\hline Views at end date & $3,317,584($ Oct 6, 2008) & 428,754 (Dec 29, 2008) \\
\hline Weeks in the Top 20 & 17 out of 29 & 29 out of 29 \\
\hline Average rank & 13 & 6 \\
\hline Ratings at end date & 4,558 & 1,391 \\
\hline Favored at end date & 4,250 & 1,636 \\
\hline Added on YouTube & July 14, 2006 & July 17, 2006 \\
\hline
\end{tabular}

the Internet [8] of Senator Stevens with music and cartoons, while Video_B contains only a picture of Senator Stevens with the audio track of his speech. Video_B is what can be called "neutral, factual information", while Video_A is an "opinionated satire".

2) Google Universal Search: The results produced by the Google Search API are not always the same with the results presented by the Google Search web interface. In fact, this discrepancy is a phenomenon already known and discussed in the literature, e.g., [9]. As an example, compare the following API results with the screenshot in Figure 2. Both are taken at the same day as a response to the query "Al Franken".

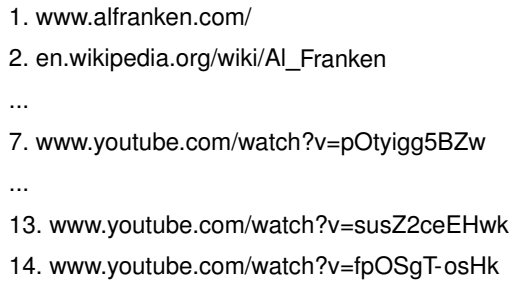

In the screenshot, two YouTube videos that in the URL list occupy positions 7 and 13, are presented side-to-side on the 4th position. Presentation of videos inside search results is part of what Google calls "the universal search model", which Google has been adopting since May 2007 [7]. However, during the data collection we noticed that the model does not apply to all queries. In fact, while Google API returns YouTube results for almost all candidates, the Google Web Interface presents universal search results for only 22 candidates. It is unknown how Google algorithms calculate which videos to show in the universal search results. We looked at the number of views, but only 18 out of the 50 most viewed videos appear in universal results. We looked at the average ranking of a video, but only 20 out of 75 videos with an average ranking between 1 and 11 (that is results that will appear in the first page of search results) appear in universal results. We looked at the content of the videos appearing in universal results and for some candidates both shown videos were positive (e.g, Kirk Schuring, Mark Warner, Ronnie Musgrove, Darcy Burner, Rick Noriega), while for some others (e.g John Cornyn, Norm Coleman, Sam Graves, Mark Udall, Gabrielle Giffords) both shown videos were negative. The lack of transparency in how videos are promoted to universal search results constitutes a technological bias that influences what kind of information users can access more easily.

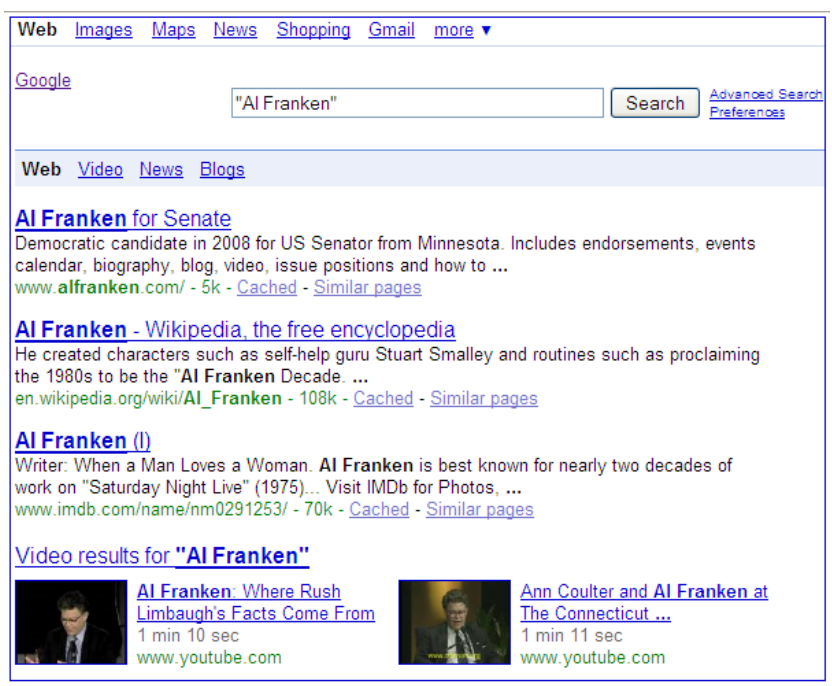

Fig. 2. Google Search results for the candidate Al Franken. Retrieved on October 28, 2008.

\section{DisCUSSION OF RELATED RESEARCH}

Search engines such as Google and YouTube give users the impression that they have access to all the information on the Web at their fingertips. However, studies (e.g. [10]) have shown that users tend to follow only results ranked highly. Consequentially, the factors taken into account by the ranking algorithms become crucial for providing the user with relevant information. Because the video medium is invisible to the search engines, the information provided around it such as number of viewers, number of comments, ratings, favorites, tags, titles, and descriptions are some of the features considered by the algorithms. Thus, the actions performed by video providers and video consumers may affect the ranking of a video in the search engine results. In this sense, YouTube may have an inherent bias.

The bias posed by YouTube has been analyzed in other contexts than politics. After finding many examples of positive 
depiction of smoking in YouTube videos, Freeman and Chapman [11] pose the important question of whether YouTube should control its content beyond protecting minors from viewing explicit content. The study by Hossler and Conroy [12] addresses this concern for tanning beds. Rather than making YouTube responsible for the lack of warning videos about tanning beds, the authors call the dermatology community to push for videos containing this message.

Our study showed that political candidates understood this sense of responsibility, since the majority of them appeared as video providers on YouTube. Similar studies from political scientists (several papers in [13]) demonstrated that politicians regard YouTube as another medium to distribute their political message, however, as not capable of mobilizing voters. In fact, the results show that most of the videos are watched less than 1,000 times and because these numbers show the global audience of a video, it is not possible to know whether voters in the district of a candidate have been watching the videos.

Another technological bias inherent to the search results is the use of the Universal Search from Google. We discovered that for only $1 / 5$ of the candidates, Google provided such results, which contained videos embedded at the top of the first results page (refer to Figure 2). While Google researchers, by using eye-tracking studies, have tried to reassure the public that the presence of videos or images does not influence the way a user perceives the search results [14], third-party investigators using the same eye-tracking technology have confirmed the contrary [15], that is, users tend to see first video and image results.

\section{CONCLUSions AND Future Work}

Although more and more Americans are turning to the Web for unfiltered information, the content analysis of 366 videosappearing in the top 20 Google Search results over a 6 month period-showed that less than $10 \%$ of the videos have a nonpartisan message. All the other videos are either pro-candidate or against-candidate. Furthermore, the political campaigns and other political groups with a stake at the election outcome have contributed $80 \%$ of videos. Thus, users should be aware that the Web is not a place free of political bias, where original, factual, non-partisan content is plenty. In fact, $57 \%$ of videos are made-for-TV political ads that have been uploaded on YouTube with the goal to reach more people.

Adding to the political bias is an inherent technological bias that is related to how YouTube and Google calculate the relevance of a video. In comparing two videos about the same event, added on YouTube at the same time, we were not able to understand why the more watched video dissapeared from the top 20. We also discovered that videos added on YouTube in September and October did appear 2 weeks earlier in the top 20 Google search results than those added in June through August. Finally, the sporadic use of Google Universal Search (videos embedded directly in the first page of results) offers access to some videos more easily than others, without a known reason. We think that such aspects of technological bias should be a topic of discussion that merits wide attention.
We have identified several areas for future research. The first one is to collaborate with political scientists to collect other queries that might be of interest to follow during an election season, beyond simply the names of the candidates. The second one is the assessment of comments and discussions surrounding a video. We noticed that although YouTube provides the means for commenting, only a very small percentage of the viewers leave a comment or engage in discussions in YouTube. We assume that while the videos are stored in YouTube, the conversations about them might be taking place in other websites that link to the videos, websites that usually specialize in political discussion. So the next step will be to track these websites (YouTube provides this possibility) and to crawl their content, in order to discover whether that is the case. Finally, we are interested in performing machine-based sentiment analysis [16] on the content of video comments, based on the insights gained from our manual analysis.

\section{ACKNOWLEDGMENT}

The authors are very grateful to the students Jess Chung, Rebecca Graber, and Morgen Maroney for their valuable contribution in the data gathering and evalution processes.

\section{REFERENCES}

[1] Online Report, The Internet and the 2008 Election, http://www. pewinternet.org/Reports/2008/The-Internet-and-the-2008-Election.aspx

[2] _, comScore Releases February 2009 U.S. Search Engine Rankings, March 13, 2009. http://www.comscore.com/Press_Events/Press_Releases/ 2009/3/US_Search_Engine_Ranking/

[3] F. Rich, 2006: The year of the 'Macaca', New York Times, November 12, 2006. http://select.nytimes.com/2006/11/12/opinion/12rich.html

[4] T. McNichol, Engineering google results to make a point, New York Times, January 22, 2004. http://www.nytimes.com/2004/01/22/ technology/circuits/22goog.html

[5] T. Zeller Jr., Gaming the search engine, in a political season, New York Times, November 6, 2006. http://www.nytimes.com/2006/11/06/business/ media/06link.html

[6] P. Metaxas and E. Mustafaraj, The battle for the 2008 US Congressional Elections on the Web. Proc. of WebSci-09: Society On-Line, March 1820, 2009, Athens, Greece.

[7] Google Press Center, Google Begins Move to Universal Search, May 16, 2007. http://www.google.com/intl/en/press/pressrel/universalsearch_ 20070516.html

[8] Series of tubes. http://en.wikipedia.org/wiki/Series $\backslash$ of $\backslash$ tubes

[9] F. McCown and M. L. Nelson, Agreeing to disagree: search engines and their public interfaces. Proc. of the 7th ACM/IEEE-CS joint conference on Digital libraries, 309-318, 2007.

[10] E. Agichtein, E. Brill, S. Dumais, R. Pagno, Learning user interaction models for predicting web search result preferences Proc. of the 29th ACM SIGIR conference, 3-10, 2006.

[11] B. Freeman, S. Chapman Is YouTube telling or selling you something? Tobacco content on the YouTube video-sharing website. In British Medical Journal: Tobbaco Control, 16, 207-210, 2007.

[12] E. Hossler, M. Conroy, YouTube as a Source of Information on Tanning Bed Use. In Archives of Dermatology (American Medical Association), Vol 144 (No. 10), 1395-1396, 2008.

[13] S. Schulman, M. Xenos, Proceedings of YouTube and the 2008 Election Cycle in the United States. http://www.umass.edu/polsci/youtube/, University of Massachussets Amherst, April 2009.

[14] Google Blog, Eye-tracking studies: more than meets the eye. February, 2009. http://googleblog.blogspot.com/2009/02/ eye-tracking-studies-more-than-meets.html,

[15] G. Hotchkiss, Eye Tracking On Universal And Personalized Search. September, 2007. http://searchengineland.com/ eye-tracking-on-universal-and-personalized-search-12233

[16] B. Pang, L. Lee, Opinion mining and sentiment analysis. Foundations and Trends in Information Retrieval 2(1-2), pp. 1135, 2008. 